\title{
Há Controvérsia Entre Análises de Beta e Sigma-Convergência no Brasil?
}

\author{
Christiano Penna*, Fabrício Linhares ${ }^{\dagger}$
}

\author{
Conteúdo: 1. Introdução; 2. Não-Linearidades, Beta e Sigma-Convergência; 3. Modelos \\ Empíricos Não-Lineares; 4. Dados; 5. Resultados; 6. Conclusões; A. Apêndice. \\ Palavras-chave: Crescimento; Convergência; Testes Empíricos; PIBs Estaduais Per Capita; Modelos \\ Não-Lineares. \\ Códigos JEL: $\quad$ 047, 033, 011
}

O presente artigo aborda uma possível controvérsia entre testes de convergência de renda per capita com base em análises com séries de tempo e análises cross-section. As análises cross-section têm como base a hipótese da beta-convergência, enquanto as análises de tempo equivalem a análises de sigma-convergência. Aqui se argumenta que possíveis conflitos se devem a uma possível não-linearidade no processo de convergência associada à formação de clubes de convergência. Testes da hipótese de convergência com base nos modelos não-lineares de Hansen (2000) e de Phillips e Sul (2007) são conduzidos para o caso brasileiro. O primeiro teste é baseado numa regressão cross-section, enquanto o segundo, em análise de séries temporais. Ambos os testes apontam para a formação de dois clubes de convergência semelhantes. Para cada clube constatam-se evidências tanto de beta quanto de sigma convergência, o que dirimi a controvérsia de resultados reportada na literatura.

This article discusses a controversy between tests of convergence of percapita income based on time series analyses and cross-section regressions. The cross-section analyses are based on the hypothesis of beta convergence, while the second one is equivalent to sigma-convergence analysis. Here, it is argued that this conflict is due to a possible non-linearity in the convergence process associated with formation of convergence clubs. Tests of the convergence hypothesis based on nonlinear models of Hansen (2000) and Phillips e Sul (2007) are conducted for the Brazilian case. The first test is based on a cross-section regression, while the second in time series analysis. Both tests point to the formation of two convergence clubs alike. For each club is noted

*Professor do Curso de Finanças da UFC — Campus Avançado Sobral. E-mail: cmp@caen.uf c.br

$\dagger$ Professor do CAEN / UFC. E-mail: fabcarlin@yahoo.com 
evidence of both beta and sigma convergence, and the controversy of results previously reported is resolved.

\section{INTRODUÇÃO}

A literatura que investiga empiricamente a hipótese da convergência é vasta e continua se desenvolvendo. Desde a análise inicial de Wiliam Baumol, em 1986, diversas técnicas e aprimoramentos foram propostos com o intuito de se testar se a evolução da renda per capita de países ou regiões é compatível com a hipótese de convergência. ${ }^{1}$

As análises iniciais carregavam em seu bojo uma predição particular do modelo de Solow (1956), senão a principal: dado que a produtividade marginal do capital era decrescente, conforme o estoque de capital per capita das economias fosse se ampliando, então o nível das rendas per capita das economias deveria convergir para um patamar comum no longo prazo.

A predição da convergência sugeria uma dinâmica para as diversas economias que podia ser empiricamente testada e, grosso modo, este teste passou a ser realizado de três maneiras distintas:

(i) através da $\beta$-convergência;

(ii) através da $\sigma$-convergência e;

(iii) através da análise da dinâmica da intra-distribuição da renda per capita.

Os dois primeiros conceitos foram formalmente definidos no conhecido trabalho de Barro e SalaI-Martin (1991): quando a dispersão da renda real per capita entre um grupo de economias se reduz ao longo do tempo, diz-se que ocorre a $\sigma$-convergência e, quando a correlação parcial entre a taxa de crescimento da renda real per capita e o nível de renda real inicial é negativo, diz-se que há $\beta$ convergência. O terceiro conceito foi sugerido por Quah (1996), que deduz a validade da hipótese da convergência a partir de mudanças na distribuição da renda, no sentido desta se tornar mais equitativa ao longo do tempo.

Salvo a análise de Quah (1996), grande parte dos testes propostos pela literatura se concentra nos conceitos de $\beta$ e $\sigma$-convergência. Uma revisão atenta da literatura internacional sugere que os testes propostos por Abramovitz (1986), Baumol (1986), Barro e Sala-I-Martin (1991), Mankiw et alii (1992), Durlauf e Johnson (1995) e Hansen (2000), são basicamente focados em regressões cross-section e que as análises de Islam (1995), Caselli et alii (1996) e Bond et alii (2001) tem como foco o estudo do processo de convergência com base em modelos de painéis dinâmicos. Uma característica marcante destes testes é a de que todos eles são focados na constatação da relação negativa entre taxa de crescimento da renda real per capita e nível de renda real inicial, ou seja, no conceito de $\beta$-convergência.

Já os testes de Bernard e Durlauf (1995), Evans e Karras (1996), Pesaran (2007), Mello e GuimarãesFilho (2007), Phillips e Sul (2007) e Beyaert e Camacho (2008), formulados em modelos de séries temporais, investigam se a dispersão da renda real per capita entre um grupo de economias vem se reduzindo ao longo do tempo, isto é, enfatizam a hipótese de $\sigma$-convergência.

Especificamente para o caso brasileiro, os estudos de Ellery JR. e Ferreira (1994), Ferreira e Diniz (1995, 1996), Azzoni (1997, 2001), Ferreira (1999), Dos Santos e Carvalho (2007), averiguaram o processo de $\beta$-convergência absoluta e identificaram um coeficiente negativo e estatisticamente significante para a variável renda per capita inicial, o que sugere que a hipótese de convergência deve ser sustentada. Utilizando "regressões de Solow Aumentadas", os estudos de Ferreira (2000), Azzoni et alii (2000), Chaves (2003), Silveira-Neto e Azzoni (2005) e Resende e Figueiredo (2005) analisaram a ocorrência de $\beta$ convergência condicional, e também encontraram evidencias de ocorrência da mesma. Outra vertente

\footnotetext{
${ }^{1} \mathrm{O}$ trabalho de Durlauf et alii (2004) é o que parece ter reunido de forma mais abrangente as diversas análises empíricas propostas na literatura.
} 
que investiga a ocorrência da $\beta$-convergência faz uso de painéis dinâmicos. Dentre os estudos que utilizaram esta metodologia pode-se citar Trompieri Neto et alii (2008), Abitante (2007), Costa (2009), Veloso et alii (2008) e Cangussu et alii (2010).

Com relação às análises de séries temporais, referentes à hipótese da $\sigma$-convergência, poucas aplicações foram feitas para o caso brasileiro. Os principais estudos são: Barossi-Filho e Azzoni (2003), Lima et alii (2010) e Mello (2011). O primeiro trabalho fez uso de testes de raiz unitária, o segundo modelou as rendas per capitas com relação à de São Paulo com base em um modelo ARFIMA e a terceira análise utiliza testes de raiz unitária com intervalos de confiança que levam em conta a persistência e o reduzido tamanho amostral das séries. Barossi-Filho e Azzoni (2003) rejeitam a hipótese de convergência para os estados do Norte; Lima et alii (2010) lançam dúvidas a respeito da convergência para o estado de São Paulo (que é tido como benchmark devido ao fato de ser o estado que historicamente detém a renda per capita mais elevada) enquanto que Mello (2011) encontra evidências de convergência estocástica, embora este resultado não se mantenha ao se utilizar a correção de intervalo de confiança com base na maior raiz autoregressiva proposta em Stock (1991).

A apreciação geral destes estudos sugere que, de modo similar ao encontrado por Bernard e Durlauf (1995) para a literatura internacional, parece não haver um concordância na literatura empírica para o caso brasileiro em termos de resultado. Enquanto as análises cross-section (que em sua maioria são análises de $\beta$-convergência) sugerem a ocorrência de convergência, as análises de séries temporais (que, por sua vez, carregam em sua estrutura a ideia de $\sigma$-convergência) indicam a inexistência da mesma. Por sinal, esse resultado é semelhante ao constatado por Young et alii (2008) para o caso norte americano. ${ }^{2}$

Teoricamente, a conexão entre $\beta$ e $\sigma$-convergência se dá devido aos retornos decrescentes do capital. Havendo tal especificidade, então a $\beta$-convergência necessariamente deve ocorrer; a $\sigma$-convergência, entretanto, poderia ser violada devido ao que se conhece por polarização ou devido a fenômenos de curto prazo capazes de levar uma economia, ou grupos de economias, a sua posição de steady-state de modo mais acelerado do que as demais.

Assim, num contexto regional onde há livre mobilidade de capital e baixas barreiras a migração, o presente estudo se concentra na ideia de que a $\beta$-convergência deveria acompanhar a $\sigma$-convergência no longo prazo, desde que as economias compartilhem uma mesma posição de steady-state. Com efeito, aqui se argumenta que o desconforme de resultados entre estes tipos de análise de convergência deve ser revisto sob a perspectiva de que as unidades investigadas, mesmo que localizadas no mesmo país, não compartilhem um único steady-state, sendo mais apropriado então o emprego de modelos empíricos não-lineares. Isto decorre do fato de que, se não-linearidades associadas à formação de clubes de convergência forem equivocadamente negligenciadas, então há uma tendência de que as análises de $\beta$-convergência e $\sigma$-convergência surtam resultados possivelmente distintos e, talvez por conta disso, a incompatibilidade de resultados gerados pelas análises de séries de tempo e dados cross-section venha sendo constatada.

A proposta de se permitir um efeito não-linear no crescimento tem contrapartidas tanto teóricas quanto empíricas. Do lado teórico, a existência de não-linearidades pode decorrer, por exemplo, de externalidades do capital humano (Azariadis e Drazen, 1990) ou de restrições de liquidez no processo de acumulação de capital humano e físico, respectivamente (Galor e Zeira, 1993), ou a partir do processo de difusão tecnológica discutido em Parente e Prescott (1994), Barro e Sala-I-Martin (1997), Basu e Weil (1998), Lucas (2000), Howitt (2000) e Howitt e Mayer-Foulkes (2005). Do lado empírico, a estrutura nãolinear foi sugerida e constatada nas análises de Durlauf e Johnson (1995), Liu e Stengos (1999), Hansen (2000), Huang (2005), Phillips e Sul (2007) e Beyaert e Camacho (2008), por exemplo.

\footnotetext{
${ }^{2}$ Young et alii (2008) mostram "(i) why $\sigma$-convergence may not accompany $\beta$-convergence, (ii) discuss evidence of $\beta$-convergence in the United States, and (iii) use U.S. county-level data containing over 3,000 cross-sectional observations to demonstrate that $\sigma$-convergence cannot be detected at the county level across the United States, or within the large majority of the individual U.S. states considered separately. Indeed, in many cases statistically significant $\sigma$-divergence is found.".
} 
Com o intuito de investigar se a presença de não linearidades pode ser responsável pelo conflito de resultados, foram empregados dois modelos empíricos: o primeiro é o modelo de Hansen (2000), que é baseado numa regressão cross-section e que visa testar a hipótese de $\beta$-convergência; o segundo modelo não-linear é o modelo de Phillips e Sul (2007), o qual é baseado numa análise de séries temporais focada na ideia da $\sigma$-convergência.

Os resultados sugerem que, permitindo a possibilidade de formação de clubes de convergência, as análises de $\beta$-convergência e $\sigma$-convergência parecem estar de acordo uma com a outra para o caso brasileiro, o que vai de encontro à ideia comumente encontrada de que as análises de séries temporais tendem a gerar resultados que contrariam as análises cross-section.

Após esta introdução, a seção seguinte explora em detalhes a conexão entre as análises de $\sigma$ e $\beta$ convergência e de que forma não-linearidades são capazes de influenciar tal conexão. Posteriormente, os modelos de Hansen (2000) e Phillips e Sul (2007) são apresentados. Na quarta seção são apresentados os dados. Na quinta seção os resultados da aplicação destas duas metodologias são expostos e comentase brevemente a robustez dos resultados. A sexta seção conclui.

\section{NÃO-LINEARIDADES, BETA E SIGMA-CONVERGÊNCIA}

Barro e Sala-I-Martin (1991) sugerem que a $\beta$-convergência é uma condição necessária, mas não suficiente, para que ocorra $\sigma$-convergência, ou seja, $\sigma$-convergência implica $\beta$-convergência. ${ }^{3}$ Entretanto, $\sigma$-convergência é somente uma condição suficiente (mas não necessária) para a existência da $\beta$-convergência. Em resumo: $\sigma \Rightarrow \beta$, mas $\beta \nRightarrow \sigma$.

Um exemplo analítico com base na dinâmica de transição para o estado estacionário é capaz de elucidar esta questão. Suponha um modelo de crescimento neoclássico que trata de dois conjuntos de economias idênticas, a não ser devido ao caráter tecnológico. Suponha, assim como no modelo de Barro e Sala-I-Martin (1997), que a tecnologia é um pouco mais refinada para o primeiro grupo de economias quando comparada ao componente tecnológico do segundo grupo, isto é, $A_{1}>A_{2}$. Como se pode observar na Figura 1, a seguir, isto é suficiente para gerar duas posições de estado estacionário, $k_{1}^{*}>k_{2}^{*}$, no plano $\left[\left(\frac{k_{t}^{*}}{k_{t}}\right), k_{t}\right]$, o que por sua vez garantiria que a renda per capita destes grupos de economias convergiria, respectivamente, para $y_{1}^{*}$ e $y_{2}^{*}$, com $y_{1}^{*}>y_{2}^{*}$.

Quando o esquema descrito passa a ser visto no plano $\left[\left(\frac{y_{t}^{*}}{y_{t}}\right), y_{t}\right]$ tem-se, então, a essência do que se pretende capturar nos dois testes de convergência. Note-se que se os dados estão bem alinhados com a teoria, então, ao longo da dinâmica de transição e, partindo-se de uma regressão para a média, a $\beta$-convergência seria constatada, uma vez que existiria uma correlação negativa entre a taxa de crescimento da renda real per capita e o nível de renda inicial. Há, entretanto, uma violação da hipótese de $\sigma$-convergência, pois a dispersão da renda real per capita entre os dois grupos de economias não viria se reduzindo ao longo do tempo. ${ }^{4}$ Este exemplo teórico simples reforça o fato de que $\beta \nRightarrow \sigma$ e sugere um ponto de particular interesse: o de resultados possivelmente conflitantes entre estes dois tipos de análises.

No intuito de conciliar estes resultados, o que se pretende fazer aqui é testar o possível descarte dos usuais modelos lineares em prol dos modelos não-lineares. A justificativa empírica para isso seria o conhecido processo de formação de clubes de convergência. ${ }^{5}$ Note-se que se esta não linearidade de fato existir, então o mais sensato seria descartar a regressão para a média e se utilizar regressões que

\footnotetext{
${ }^{3}$ Furceri (2005) argumenta que esta relação não seria capaz de determinar claramente a relação causal entre $\sigma$ e $\beta$ convergência. $\mathrm{O}$ autor demonstra que a $\beta$-convergência só garantiria $\sigma$-convergência se $\beta$ for relativamente grande e $\operatorname{var}\left[\ln \left(Y_{t}\right)-\ln \left(Y_{t+\Upsilon}\right)\right]$ for suficientemente pequena.

${ }^{4}$ Também pode ser feita uma analogia muito próxima a esta para o modelo Schumpeteriano proposto em Howitt (2000).

${ }^{5}$ Este resultado não é novo e foi obtido nas análises de Mossi et alii (2003), Andrade et alii (2004), Laurini et alii (2005), Gondim et alii (2007), Coelho e Figueiredo (2007), Trompieri Neto et alii (2008) e Penna e Linhares (2009), por exemplo.
} 
Figura 1 - Convergência: da teoria para o empiricísmo

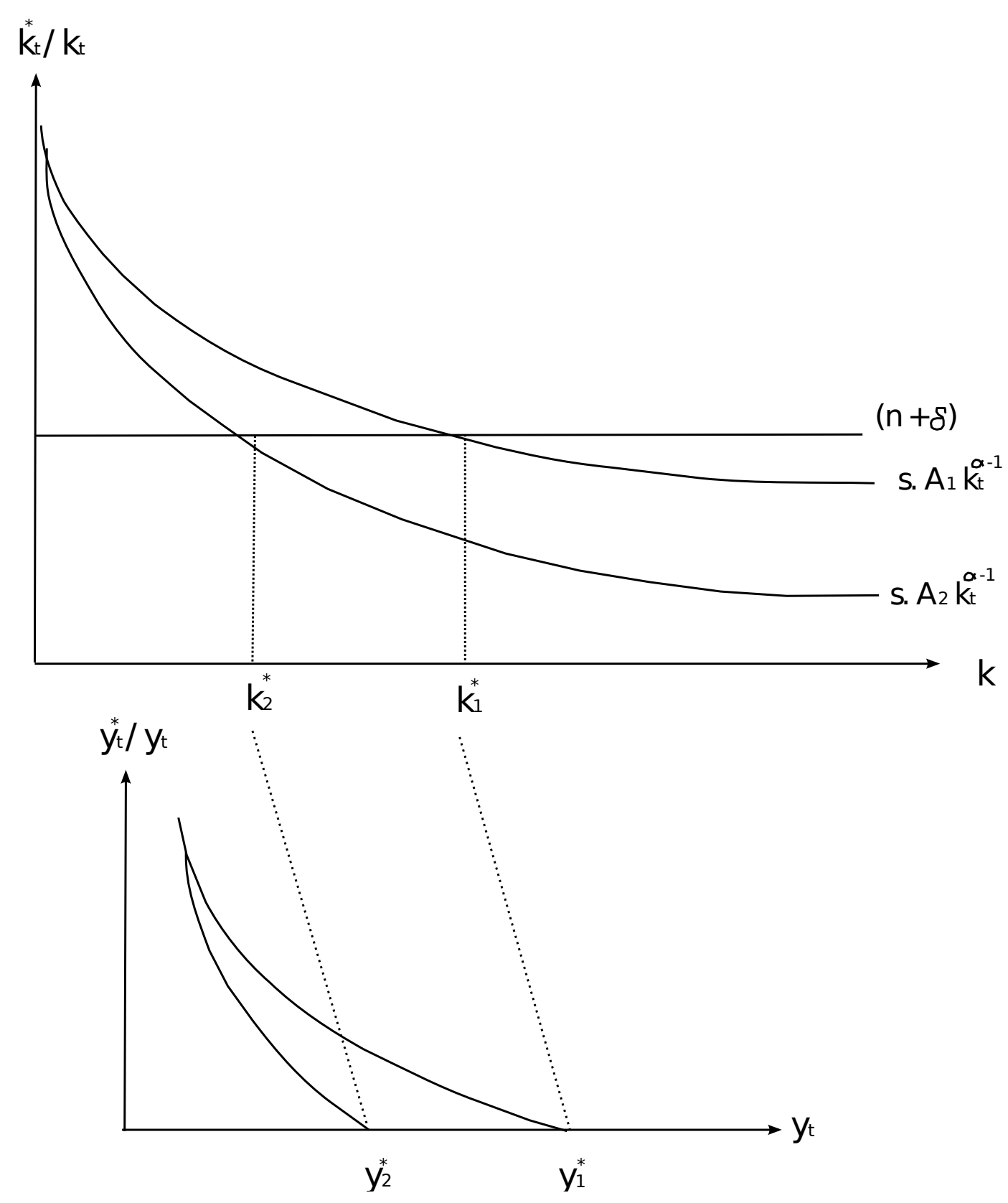


da não industrialização ou promovendo um take-off para a industrialização.

Nesta vertente também há modelos que explicam simultaneamente padrões históricos do crescimento e de outras variáveis estado associadas ao crescimento (Maddison, 2004), assim como modelos cuja transição demográfica passa a explicar os take-offs (Galor e Weil, 2000). Como tais teorias estabelecem certas condições para que as economias sejam capazes de "decolar", as economias que não cumprem estes requisitos poderiam encontrar-se presas em uma fase de estagnação econômica por longos períodos de tempo.

A segunda classe de modelos enfoca o papel do progresso tecnológico no crescimento. Estes modelos versam sobre o papel da difusão de tecnologia e tem correntes tanto neoclássicas (Barro e Sala-I-Martin, 1997) assim como endógenas (Howitt, 2000). Sob tal premissa, há também os trabalhos de Lucas (2000), Basu e Weil (1998), Parente e Prescott (1994) e Howitt e Mayer-Foulkes (2005). Nestes modelos uma atenção especial tem sido dada de forma a explorar os canais através dos quais os países menos desenvolvidos imitam ou adotam as tecnologias dos países líderes. Se não há barreiras para a difusão tecnológica entre os países, então estes modelos preveem que as rendas per capita das economias ricas e pobres iriam convergir gradualmente. No entanto, existindo barreiras, os países podem diferir na sua capacidade de adotar tecnologias de modo que se formem clubes de convergência que convergirão para níveis distintos de renda per capita. Assim sendo, as rendas per capita dos clubes de convergência não deveriam convergir e a polarização dos níveis de renda entre as economias poderia ser permanente.

Em ambos os casos, abre-se espaço para a ocorrência de formação de clubes de convergência e, como foi discutido anteriormente, o tratamento linear, ou que considera todas as economias de forma homogênea, possibilitaria uma inconsistência entre as análises de $\beta$ e $\sigma$-convergência.

\section{MODELOS EMPÍRICOS NÃO-LINEARES}

Uma das primeiras investigações empíricas formais identificadas na revisão da literatura que trata de modelos não-lineares parece ter sido proposta por Durlauf e Johnson (1995). Estes autores empregaram técnicas de Classification and Regression Tree Analysis (CART) para investigar os efeitos da renda inicial e da escolaridade no processo de convergência das economias e de seu acondicionamento em clubes de convergência. Os testes rejeitam a versão linear comumente utilizada na literatura em fa- 
vor do modelo que acomodava a possibilidade das economias se agruparem em múltiplos regimes de crescimento (ou múltiplos estados estacionários). A principal justificativa dos autores para formação de clubes de convergência foi baseada na variabilidade do produto marginal do capital com relação ao nível de desenvolvimento da economia. ${ }^{6}$

A partir daí, os trabalhos empíricos na área de crescimento parecem ter se aproveitado dos avanços estatísticos (mais especificamente, da divisão da amostra, ou "sample spliting", e de regressões com efeito limiar, ou "efeito threshold") para explorar melhor o acondicionamento dos dados aos modelos não-lineares. ${ }^{7}$ Tais técnicas tiveram início com uma série de trabalhos de Bruce Hansen e uma aplicação formal das mesmas na área de convergência pode ser vista em Hansen (2000). O autor utilizou a mesma base de dados de Durlauf e Johnson (1995) e, através de um modelo econométrico com efeito threshold condicionado ao nível de renda per capita, encontrou indícios de que as economias tendem a formar clubes de convergência cujas características dos membros são semelhantes. ${ }^{8}$

É necessário salientar que a usual regressão linear não permite uma maior inferência em relação a possíveis diferenças entre estados estacionários. O modelo com efeito threshold parece suprir esta carência devido ao fato de permitir diferentes interceptos e velocidades de convergência. Ressalte-se que, assim como no modelo linear, a abordagem de Hansen (2000) não permite inferências em favor dos fenômenos de catch-up e de pushing back. Isto decorre do fato de esta técnica ser uma análise com base numa regressão cross-section parcialmente estática e, por consequência, incapaz de capturar processos dinâmicos desse tipo.

Já a metodologia de séries temporais proposta em Phillips e Sul (2007) emprega a ideia discutida nos modelos de Barro e Sala-I-Martin (1997) e de Howitt (2000), e ganha força nas teorias apresentadas em Parente e Prescott (1994), Basu e Weil (1998), Lucas (2002) e, recentemente, em Howitt e MayerFoulkes (2005). Phillips e Sul (2007) formularam uma metodologia empírica para dados em painéis capaz de acomodar a possibilidade conjunta de diversos tipos de heterogeneidade entre as economias e, em particular, a hipótese de heterogeneidade no progresso tecnológico. Este novo método de estudo opera sob a hipótese de que o aprendizado tecnológico pode ser diferente ao longo do tempo e entre as economias e é baseado na formulação de um modelo de fatores não-lineares composto de um elemento idiossincrático variante no tempo, apropriado para mensurar os efeitos individuais de transição para o estado estacionário, e de um fator de tendência estocástica comum, que captura os efeitos da tecnologia comum.

Tal metodologia se adéqua as questões da dinâmica relativa e dos fenômenos de catch-up e pushing back discutidas em Quah (1996), permitindo ainda a análise de uma série de possibilidades de trajetórias de transição para um estado estacionário condicionado, única e exclusivamente, aos dados de renda per capita (ao contrário da proposta de Quah, esta técnica não requer matrizes de transição de Markov). Dadas tais trajetórias, e supondo que as economias compartilham de um padrão de crescimento comum, Phillips e Sul (2007) sugerem também um teste estatístico, o teste $\log t$, que ganha poder ao ser aplicado recursivamente sobre as unidades transversais, possibilitando assim, a seleção adequada de uma amostra de economias que representem um determinado clube de convergência.

\footnotetext{
${ }^{6}$ Coelho e Figueiredo (2007) aplicam a metodologia CART (nos moldes de Johnson e Takeyama, 2003) aos dados municipais brasileiros e identificaram dois clubes de convergência: um clube de baixa renda, formado predominantemente pelos municípios das regiões norte e nordeste, e outro clube de convergência com nível de renda mais elevado, composto basicamente pelos municípios das regiões centro-oeste, sudeste e sul. Os autores concluem que o surgimento destes clubes se deve, em grande parte, às desigualdades existentes entre as regiões e não dentro das regiões.

${ }^{7}$ Há também abordagens semi-paramétricas que permite não-linearidades de crescimento como, por exemplo, a discutida em Liu e Stengos (1999).

${ }^{8}$ A metodologia proposta em Hansen (2000) pode ser entendida como uma generalização da técnica de CART empregada por Durlauf e Johnson (1995) e os resultados obtidos nestes trabalhos foram bastante similares.
} 


\subsection{A Metodologia de Hansen (2000)}

A ideia central de Hansen (2000) é a de que é possível dividir a amostra com base numa função indicadora que utiliza variáveis observáveis, definidas previamente como determinantes na formação de subgrupos amostrais. Para facilitar a exposição, se parte da hipótese que há apenas dois regimes. A partir da equação de regressão cross-section sugerida em Baumol (1986),

$$
\left(\frac{1}{T}\right) \ln \left(\frac{y_{i, t_{0}+T}}{y_{i, t_{0}}}\right)=\alpha+\alpha_{1} \ln \left(y_{i, t_{0}}\right)+\varepsilon_{i}
$$

constrói-se um modelo com efeito threshold, que pode ser descrito como:

$$
\left(\frac{1}{T}\right) \ln \left(\frac{y_{i, t_{0}+T}}{y_{i, t_{0}}}\right)_{i}=\theta_{1}+\ln \left(y_{i, t_{0}}\right)_{i} I_{i}\left\{z_{i} \leq \lambda\right\}+\theta_{2} \ln \left(y_{i, t_{0}}\right)_{i} I_{2}\left\{z_{i}>\lambda\right\}+\varepsilon_{i}
$$

onde $\theta\left[\alpha, \alpha_{1}\right], I_{j}\{$.$\} é a função indicadora, com I_{1}=1$ quando $z_{i} \leq \lambda$ e zero de outra forma $I_{2}=1$ e quando $z_{i}>\lambda$ e 0 de outra forma, $z_{i}$ é a variável threshold, $\lambda$ é o parâmetro threshold e o erro $\varepsilon_{i t}$ é tido como independente e identicamente distribuído $\left(\mathrm{iid}\right.$ ) com média zero e variância finita, $\sigma^{2}<\infty$. A equação acima permite a estimação de coeficientes distintos para cada grupo da amostra delimitado pelo valor da variável threshold, notando-se que o modelo linear é um caso particular do modelo com efeito threshold com $\theta_{1}=\theta_{2}$.

Seja $\theta=\left(\theta_{1}^{\prime}, \theta_{2}^{\prime}\right)$ e $\hat{X}_{i}(\lambda)=\left(\ln \left(y_{i, t_{0}}\right)_{i}^{\prime} I_{i}, \ln \left(y_{i, t_{0}}\right)_{i}^{\prime} I_{2}\right)^{\prime}$, define-se o espaço $\wedge=[\underline{\lambda}, \bar{\lambda}]$, onde $\underline{\lambda}>\min \left\{z_{i}\right\}$ e $\bar{\lambda}<\max \left\{z_{i}\right\}$. Observe que, para cada valor de $\lambda \in \wedge$, o vetor $\hat{X}_{i}(\lambda)$ assumirá uma forma particular. A estimação de modelos com efeito threshold para dados cross-section segue a metodologia proposta em Hansen (2000), a qual procede da seguinte maneira: para cada $\lambda_{n} \in \wedge$ obtêm-se por MQO as estimativas $\hat{\alpha}_{i}\left(\lambda_{n}\right)$ e $\hat{\theta}\left(\lambda_{n}\right)$ e o respectivo somatório do quadrado dos resíduos $S\left(\lambda_{n}\right)=\sum \sum \hat{\varepsilon}_{i t}^{2}\left(\lambda_{n}\right)$, e as estimativas finais de $\alpha_{i}, \theta$ e $\lambda$ são os valores que minimizam a função $S\left(\lambda_{n}\right)$.

O teste para existência de efeito limiar, $\theta_{1} \neq \theta_{2}$, utiliza a estatística de teste de multiplicador de Lagrange que é robusta à heterocedasticidade e tem seus valores críticos determinados por um procedimento de bootstrap (Hansen, 1996). Caso o efeito limiar seja estatisticamente relevante na equação (2), conclui-se que existem pelo menos dois regimes, nos quais as relações entre a variável dependente e as variáveis explicativas são distintas (ao menos para algumas delas). Do contrário, o modelo mais adequado para os dados é dado pela equação (1), ou seja, o modelo linear inicialmente proposto por Baumol (1986).

\subsection{A Metodologia de Phillips e Sul (2007)}

Seja $X_{i t}$ o PIB per capita da $i$-ésima unidade cross-section no tempo $t$, onde $i=1, \ldots, N$ e $t=$ $1, \ldots, T$. Usualmente, $X_{i, t}$ é decomposto em duas partes, uma sistemática, $g_{i, t}$, que inclui elementos comuns às unidades cross-section, e uma transitória, $a_{i, t}$, ou seja, $X_{i, t}=g_{i, t}+a_{i, t}$. Observe que $g_{i, t}$ e $a_{i, t}$ podem ser formados por uma mistura de componentes comuns e idiossincráticos. A estratégia empírica de Phillips e Sul (2007) é modelar $X_{i, t}$ de modo que a parte comum seja separada da parte idiossincrática, ou seja,

$$
X_{i, t}=g_{i, t}+a_{i, t}=\left(\frac{g_{i, t}+a_{i, t}}{\mu_{t}}\right) \mu_{t}=b_{i, t} \mu_{t}
$$

onde $\mu_{t}$ é um componente que determina a trajetória de longo prazo, ou seja, uma trajetória comum de crescimento do PIB per capita e $b_{i, t}$ é um elemento idiossincrático, que varia no tempo, capaz de mensurar a transição individual de $i$, dado o seu deslocamento em torno da trajetória comum, $\mu_{t}$.

Nestes termos, seria possível se testar convergência de longo prazo (quando $t \rightarrow \infty$ ) sempre que a heterogeneidade não observada se dissipe, ou seja, sempre que $g_{i, t} \rightarrow g_{i}$. Neste caso, pode-se ter 
convergência global se $b_{i, t} \rightarrow b$, ou formação de clubes de convergência, isto é, $b_{k, t} \rightarrow b_{k} \& b_{j, t} \rightarrow$ $b_{j}, k \neq j$. Inferências sobre o comportamento de $b_{i, t}$ não são possíveis sem a imposição de alguma restrição em sua dinâmica, pois o número de parâmetros desconhecidos em $b_{i, t}$ é igual ao número de observações; uma alternativa para modelar os elementos de transição, $b_{i, t}$, vem da construção de um coeficiente de transição relativo, $h_{i, t}$, definido como: $h_{i, t}=\frac{\hat{x}_{i, t}}{\left(N^{-1} \sum_{i=1}^{N} \hat{x}_{i, t}\right)}=\frac{b_{i, t}}{\left(N^{-1} \sum_{i=1}^{N} b_{i, t}\right)}$, onde $\hat{x}_{i, t}$ representa o PIB per capita sem o componente de ciclos econômicos. ${ }^{9}$

Sendo assim, as curvas traçadas por $h_{i, t}$ definem uma trajetória de transição relativa e, ao mesmo tempo, mensuram quanto o PIB per capita $i$ se desloca em relação à trajetória de crescimento comum, $\mu_{t}$. Dessa forma, $h_{i, t}$ pode diferir entre as $i$ economias no curto prazo, mas admite convergência no longo prazo sempre que $h_{i, t} \rightarrow 1$ para todo $i$ quando $t \rightarrow \infty$. Ressalta-se ainda que, se isso ocorrer, no longo prazo a variância cross-section de converge para zero; ou seja, tem se que: $\sigma_{t}^{2}=N^{-1} \sum_{i=1}^{N}\left(h_{i, t}-1\right)^{2}$ quando $t \rightarrow \infty$, que é a base da $\beta$-convergência.

Com base nesta modelagem, Phillips e Sul (2007) desenvolveram uma análise de convergência baseada no que denominaram teste $\log t$. Os autores propõem uma forma semi-paramétrica para modelar os coeficientes de transição assumindo que os mesmos são tendências estocásticas lineares e permitindo heterogeneidade entre economias ao longo do tempo: $b_{i, t}=b_{i}+\frac{\sigma_{i} \xi_{i, t}}{L(t) t^{a l p h a}}$, onde $L(t)$ é uma função slowly varying, crescente e divergente no infinito; ${ }^{10} \xi_{i, t} \sim$ i.i.d $(0,1), \alpha$ governa a taxa de queda da variação nas unidades transversais ao longo do tempo e, $\sigma_{i}>0$ e $t \geq 1, \forall i$.

Notando que, $L(t) \rightarrow \infty$ quando $t \rightarrow \infty$, então essa formulação assegura que para todo $\alpha \geq 0$, ou seja, sempre haverá convergência se $b_{i, t} \rightarrow b_{i}$ para todo $\alpha \geq 0$ e divergência caso contrário. Com efeito, têm-se duas condições para convergência do modelo:

1. $\lim _{k \rightarrow \infty} b_{i, t+k}=b \Leftrightarrow b_{i}=b$ e $\alpha \geq 0$ e

2. $\lim _{k \rightarrow \infty} b_{i, t+k}=b \Leftrightarrow b_{i} \neq b$ ou $\alpha<0$,

Ou seja, é possível estabelecer um teste da hipótese nula de convergência contra hipóteses alternativas de não-convergência. Tal teste é baseado nas seguintes hipóteses:

$$
\begin{array}{ll}
\text { Hipótese nula } & H_{0}: b_{i}=b \text { e } \alpha \geq 0 \\
\text { Hipóteses alternativas } & \left\{\begin{array}{l}
H_{A 1}: b_{i}=b, \forall i \text { e } \alpha<0 \\
H_{A 2}: b_{i} \neq b, \text { para algum } i \text { e } \alpha \geq 0 \text { ou } \alpha<0
\end{array}\right.
\end{array}
$$

Tal abordagem também permite testar a formação de clubes de convergência. Por exemplo, existindo dois clubes $\left\{G_{1}, G_{2}\right\} ; G_{1}+G_{2}=N$ então a hipótese alternativa pode ser descrita da seguinte maneira:

$$
H_{A}: b_{i t} \rightarrow\left\{\begin{array}{l}
b_{1} \text { e } \alpha \geq 0 \text { se } i \in G_{1} \\
b_{2} \text { e } \alpha \geq 0 \text { se } i \in G_{2}
\end{array}\right.
$$

A regressão para se testar $b_{i, t}=b_{i}+\frac{\sigma_{i} \xi_{i, t}}{L(t) t^{\alpha}}$ supondo $L(t)=\log t$ é baseada na seguinte regressão:

$$
\log \left(\frac{H_{1}}{H_{t}}\right)-2 \log [L(t)]=\pi_{0}+\pi_{1} \log t+\mu_{t} \text { para } t=T_{0}, \ldots, T
$$

\footnotetext{
${ }^{9} \mathrm{Na}$ prática, a variável utilizada pode ser descrita como $\log y_{i, t}=b_{i, t} \cdot \mu_{t}+k_{i, t}$, onde $k_{i, t}$ representa um efeito de ciclo de negócios. A remoção do componente de ciclos pode ser realizada através da utilização do filtro de Whittaker-HodrickPrescott (WHP). Esta abordagem não requer nenhuma especificação a priori para $\mu_{t}$ e é bastante cômoda, pois requer um único parâmetro de smooth como input.

${ }^{10}$ A função $L(t)$ é dita slowly varying desde que $L(t) t^{\alpha} \rightarrow \infty$ quando $t \rightarrow \infty$ e $\alpha>0$. Note que a tendência para o infinito suavemente garante que, neste caso, $b_{i, t} \rightarrow b_{i}$.
} 
onde $\frac{H_{1}}{H_{t}}$ representa a relação de variância cross-section encontrada através de $H_{t}=N^{-1} \sum_{i=1}^{N}\left(h_{i t}-\right.$ $1)^{2}$ e $h_{i t}=\frac{\hat{w}_{i t}}{N^{-1} \sum_{i=1}^{N} \hat{w}_{i t}}$, sendo que representa o logaritmo neperiano da renda per capita do estado $i$ após a remoção do componente de ciclos através do filtro de Hodrick-Prescott.

Sob hipótese nula, os coeficientes de (4) podem ser testados com base num teste $t$ unilateral, robusto a auto-correlação e heterocedasticidade. Para um nível de 5\%, por exemplo, a hipótese nula de convergência deve ser rejeitada se $t_{\hat{\pi}_{1}}<-1,65$.

Os autores revelam que $\hat{\pi}_{1}=2 \hat{\alpha}$, onde $\hat{\alpha}$ é a estimativa de $\alpha$ sob hipótese nula. Phillips e Sul (2007) também sugerem que, para eliminar o efeito das observações iniciais, a regressão (4) seja realizada após se descartar uma determinada fração amostral. ${ }^{11}$

A rejeição da hipótese nula de convergência para todo o painel pode estar indicando a existência de pontos separados de equilíbrio ou múltiplos estados estacionários. Quando isso ocorre, pode-se ter a divergência de alguns membros do painel e/ou a formação de clubes de convergência. Neste contexto, um algoritmo que aplique sequencialmente o teste $\log t$ permite a identificação de clubes de convergência sem que se recorra às usuais características observáveis que condicionem o devido agrupamento deste clube. $O$ algoritmo é descrito a seguir:

1. Ordenam-se os PIB per capitas de acordo com o período final;

2. Selecionam-se as $k<N$ primeiras economias com maior PIB per capita, formando um grupo $G_{k}$. Estima-se a regressão $\log t$ e calcula-se a estatística de convergência, $t_{k}$, para este grupo. Escolhese um grupo formado por $k^{*}$ economias tal que a estatística de teste seja maximizada sobre $k$ de acordo com a condição: $k^{*}=\arg \max \left\{t_{k}\right\}$ sujeito a $\min \left\{t_{k}\right\}{ }^{12}$ Se a condição $\min \left\{t_{k}\right\}>-1,65$ não for válida para $k=2$, então a economia com maior PIB per capita é excluída da amostra e um novo grupo é formado. Repete-se este passo computando-se a estatística $t$ para este novo grupo e, se a condição $\min \left\{t_{k}\right\}>-1,65$ não for válida para todos os pares sequenciais de PIBs per capita, conclui-se que o painel não apresenta clubes de convergência.

3. Adiciona-se uma economia por vez ao grupo primário com $k^{*}$ membros e estima-se a regressão $\log t$ novamente; sempre se inclui uma nova economia ao clube de convergência se a estatística $t$ for maior do que o critério de fixação, $c^{*}$. Quando $T$ for pequeno, o critério de fixação, $c^{*}$, pode ser zero para assegurar uma seleção conservadora; se $T$ for grande, $c^{*}$ pode ir assintóticamente para o valor crítico de $5 \%$, ou seja, $-1,65$. Repete-se esse procedimento para todas as economias remanescentes e forma-se o primeiro sub-grupo de convergência a partir do grupo primário $G_{k^{*}}$ suplementado pelas economias que atendem ao critério de fixação.

4. Forma-se um segundo grupo com as economias cuja regra de fixação falha no passo (3); estima-se a regressão $\log t$ e se verifica se $t_{\hat{p} i}>-1,65$, que retrata o nível de significância do teste para a convergência. Se esta condição for atendida conclui-se que existem dois grupos de convergência distintos: o grupo primário $G_{k^{*}}$ e o segundo grupo. De modo contrário, se a condição não for atendida, repete-se do passo (1) ao passo (3) para verificar se este segundo grupo pode ser subdividido em um número maior de clubes de convergência. Não existindo um conjunto composto por $k \geq 2$ economias no passo (2) com $t_{k}>-1,65$, conclui-se que as economias remanescentes não podem ser subdivididas em subgrupos e, portanto, não convergem para um patamar comum.

\footnotetext{
${ }^{11}$ Estes pontos são de extrema importância para que se chegue a resultados robustos. Voltaremos a discutir os mesmos após apresentar nossos resultados.

${ }^{12} \mathrm{~A}$ condição retrata o nível de significância da análise, 5\%.
} 


\section{DADOS}

A história recente do Brasil impõe alguns percalços às análises baseadas em séries temporais estaduais. O estado do Tocantins e do Mato Grosso do Sul, por exemplo, são estados de emancipação recente (1988 e 1977, respectivamente) e, devido a isto, há pouca ou nenhuma informação disponível para períodos anteriores às suas criações. Assim, se o pesquisador está interessado em formular um painel contendo a renda per capita de todos os estados, esta indisponibilidade de dados não permite que se tome um período de tempo tão longo o quanto se queira.

Neste sentido, existem duas propostas para lidar com os dados. A primeira delas, e a mais usual, foi adotada por Azzoni (1997): ${ }^{13}$ a estratégia é manter a estrutura geopolítica vigente em um determinado período precedente à emancipação dos estados. Em se tratando do período 1970-2008, por exemplo, a sugestão é a de que se agregue o estado do Tocantins ao de Goiás, agregar o estado do Mato Grosso do Sul ao estado do Mato Grosso e, também reunir os estados do Acre, Rondônia e Roraima ao estado do Amazonas. A desvantagem desta proposta é deixar de lado a possibilidade de análises específicas em relação aos estados que estejam sendo agregados.

Uma segunda proposta seria empregar técnicas de séries temporais para tentar descrever de que forma os PIBs ou os PIBs per capita destes estados evoluíram antes de suas fundações. A partir desta proposta, Penna e Linhares (2009) realizaram uma "previsão para o passado" dos PIBs com base num processo auto-regressivo de primeira ordem: como não existem dados para o período pré-fundação dos estados do Acre, Amapá, Mato Grosso do Sul, Roraima e Rondônia e Tocantins, os autores invertem a cronologia dos dados dentro do período em análise, realizam uma previsão dinâmica e preenchem as séries de PIB per capita invertendo novamente a cronologia dos pontos previstos.

Um problema com esta segunda proposta é o de que os resultados dos testes ficam condicionados à maneira com se está buscando projetar os dados passados, além das propriedades estatísticas destas previsões. Como não se sabe ao certo quais os verdadeiros valores dos PIBs e das populações para os estados com formação recente, toda e qualquer maneira de se antever tais valores com base em propostas do tipo poderia gerar resultados no mínimo frágeis.

Posto isto, torna-se condizente trabalhar com uma base de dados que siga a primeira proposta supracitada. Esta base de dados é focada nos dados de Azzoni (1997). Os dados foram inicialmente construídos com base na arrecadação do ICMS para obter uma aproximação da participação relativa dos PIBs estaduais no PIB total e aborda o período de 1944 até os anos recentes. Ferreira (1998), entretanto, argumenta que os dados anteriores a 1970 podem não ser muito confiáveis e, seguindo tal argumento, optou-se aqui por restringir a análise ao período 1970-2008.

Ao se comparar as proporções relativas do PIB com base na metodologia de Azzoni e com base nos dados do IPEADATA para o período 1985-2008, são observadas algumas discrepâncias. Como é de se esperar que os dados do IPEA sejam mais fidedignos que os sugeridos por Azzoni, também se utilizou para os períodos mais recentes (1985-2008) as participações relativas sugeridas pelo IPEA.

Posteriormente agrega-se o PIB do estado do Tocantins ao de Goiás, o PIB do Mato Grosso do Sul ao do Mato Grosso e também os PIBs dos estados do Acre, de Rondônia e de Roraima ao PIB do estado do Amazonas. Dividindo-se estes PIBs pela soma das populações destas regiões, encontram-se, então, os PIBs per capitas destas unidades. Os PIBs per capitas dos demais estados (que não sofreram alterações em suas estruturas geopolíticas) são facilmente encontrados no IPEADATA. Todos os dados foram convertidos para R\$ de 2009.

Embora a proposta de Hansen (2000) seja uma alternativa atraente para se analisar a hipótese de $\beta$ convergência condicional, o pesquisador que pretende implementar tal teste para o caso brasileiro depara-se com um dilema, pois é necessário um número de unidades cross-section relativamente grande, o que torna difícil a aplicação direta do teste devido às poucas unidades federativas brasileiras. ${ }^{14}$ Com

\footnotetext{
${ }^{13}$ Análises deste tipo podem ser vistas em Azzoni (1997), em Costa (2009), em Cabral (2008) e, em Lima et alii (2010).

${ }^{14}$ A base de dados utilizada por Hansen (2000), por exemplo, trata de 98 unidades transversais.
} 
objetivo de se manter a análise da $\beta$-convergência em consonância com a análise da $\sigma$-convergência, isto é, buscando conectar o resultado do teste de Hansen (2000) com os resultados obtidos a partir do teste de Phillips e Sul (2007), a mesma base de dados utilizada anteriormente foi mantida.

Dado o reduzido número de seções transversais, uma maneira conveniente de se buscar um maior número de observações parece ser a de se trabalhar com um pool de dados, ou dados agrupados. A proposta de se utilizar pools é recorrente na literatura e pode ser vista em Liu e Stengos (1999), Ferreira (2000), Resende e Figueiredo (2005) e Costa (2009), por exemplo.

Em posse do painel de dados para as 20 unidades cross-section para o período 1970-2008, o pool foi construído levando-se em conta a taxa média de crescimento entre 1970-2003, 1971-2004, 1972-2005, 1973-2006, 1974-2007, 1975-2008 e as respectivas rendas per capitas iniciais (1970, 1971, 1972, ... 1975). A ideia desta construção é a de se trazer alguma dinâmica para a análise, além disso, é de se esperar que a dimensão temporal de 33 anos se adéque bem para capturar os efeitos de longo prazo e que dimensão de unidades cross-section seja suficientemente grande para que se prossiga com as estimações do teste de Hansen (2000). Tem-se, então, 120 observações de taxa média de crescimento e renda per capita inicial, ou seja, seis para cada estado em questão. A Figura 3, no Apêndice, plota as taxa médias de crescimento e a renda per capita inicial dos estados. A observação do gráfico já adianta alguns padrões que serão discutidos em detalhes mais adiante.

\section{RESULTADOS}

A seguir são expostos os resultados da aplicação dos testes de Hansen (2000) e de Phillips e Sul (2007) aos dados estaduais supracitados.

\subsection{Hansen (2000)}

Os resultados apresentados a seguir são fruto da aplicação do teste proposto por Hansen (2000) ao referido pool de dados. Inicialmente, estima-se um modelo linear, cuja estrutura é semelhante à proposta de Baumol (1986) e, posteriormente, testa-se o grau de ajuste deste modelo quando comparado ao modelo com efeito threshold. As estimativas do modelo linear são as que se seguem na Tabela 1.

Note-se que, no modelo de Baumol (1986) o efeito da renda inicial na taxa média de crescimento $(-0,00805)$ é negativo e estatisticamente significante, conforme sugere a teoria.

Tabela 1: O teste linear de Baumol (1986) — 1970-2008

\begin{tabular}{lrccc}
\hline$\left(\frac{1}{33}\right) \ln \left(\frac{y_{i, t}}{y_{i, t-33}}\right)=\alpha+\alpha_{1} \ln \left(y_{i, t-33}\right)+\varepsilon_{i} t=2003, \ldots, 2008$ \\
\hline Parâmetro & Coeficiente & Erro Padrão & Estatística $t$ & $R^{2}$ \\
\hline$\alpha$ & 0,09174 & 0,00558 & 16,45 & 0,3681 \\
$\alpha_{1}$ & $-0,00805$ & 0,00065 & $-12,29$ & \\
\hline
\end{tabular}

Passando-se para a análise do modelo não-linear, a proposta de Hansen (2000) é a de que se teste o grau de ajuste do modelo estimado de Baumol frente à especificação com dois ou mais regimes. Hansen (1996) sugere que este teste seja feito com base na estatística do multiplicador de Lagrange, a qual retorna estatísticas de testes com valor máximo de 30.360 cujo $p$-valor é de 0,000 . $0 p$-valor é obtido através de bootstrap com base em 1000 replicações, e sugere que a hipótese nula de linearidade, 
ou ausência de efeito threshold, deve ser rejeitada. Com efeito, tem-se evidência de que o modelo de Baumol (1986) deve ser descartado em favor do modelo com dois regimes. ${ }^{15}$

O parâmetro threshold foi estimado em 8,5035; este valor se refere ao logaritmo neperiano da renda per capita inicial e condiz com uma renda per capita equivalente à $\mathrm{R} \$ 4.931,79$ (em $\mathrm{R} \$$ de 2009). Este valor é o que minimiza a soma dos quadrados dos resíduos da regressão, ou a razão de máxima verossimilhança. Tal parâmetro segrega o pool de dados em dois regimes: o primeiro regime é formado por 62 das 120 observações e atende a condição $\ln \left(y_{i, t_{0}}\right)>\lambda$, ou seja, este regime é formado pelas observações cuja renda per capita inicial é superior aos $\mathrm{R} \$ 4.931,79$. O segundo regime é formado pelas observações cuja renda per capita é igual ou inferior ao referido patamar.

No intuito de identificar os estados que participam de cada regime no pool, propõe-se que a média dos logaritmos da renda per capita dos seis anos iniciais seja tomada e comparada com o parâmetro threshold. Isto evita que se contamine a análise por meio das rendas per capitas iniciais mais recentes do pool e de eventuais desvios de curto prazo. Assim fazendo, os regimes ficam formados pelos seguintes estados:

- REGIME I: $\Leftrightarrow \ln \left(y_{i, t_{0}}\right)>\lambda$

Região Amazônica (AM + AC + RO + RR), Bahia, Espírito Santo, Goiás + Tocantins, Mato Grosso + Mato Grosso do Sul, Minas Gerais, Paraná, Rio de Janeiro, Rio Grande do Sul, Santa Catarina e São Paulo.

- REGIME II $\Leftrightarrow \ln \left(y_{i, t_{0}}\right) \leq \lambda$

Alagoas, Ceará, Maranhão, Pará+Amapá, Paraíba, Pernambuco, Piauí, Rio grande do Norte e Sergipe.

As estimativas para estes dois regimes vêm a seguir, na Tabela 2:

Tabela 2: O teste de Hansen (2000) - 1970-2008

\begin{tabular}{clrrrr}
\hline$\left(\frac{1}{33}\right) \ln \left(\frac{y_{i, t}}{y_{i, t 0}}\right)=\left[\alpha+\alpha_{1} \ln \left(y_{i, t 0}\right)\right] I_{\left\{\ln y_{i, t 0}>\lambda\right\}}+\left[\alpha^{I} \alpha_{1}^{I I} \ln \left(y_{i, t 0}\right)\right] I_{\left\{\ln \left(y_{i, t 0}\right) \leq \lambda\right\}}+\varepsilon_{i}$ \\
\hline Regime & Parâmetro & Coeficiente & Erro Padrão & Estatística $t$ & $R^{2}$ \\
\hline \multirow{2}{*}{$I \Leftarrow \ln \left(y_{i, t 0}\right)>\lambda$} & $\alpha$ & 0,17231 & 0,01215 & 14,18 & 0,6649 \\
& $\alpha_{1}$ & $-0,01677$ & 0,00129 & $-13,05$ & \\
\hline \multirow{2}{*}{$I \Leftarrow \ln \left(y_{i, t 0}\right) \leq \lambda$} & $\alpha$ & 0,10030 & 0,01841 & 5,45 & 0,2209 \\
& $\alpha$ & $-0,00936$ & 0,00234 & $-4,00$ & \\
\hline
\end{tabular}

Fonte: Elaboração do autor.

o $R^{2}$ conjunto para o modelo threshold foi de 0,5513 , o que sugere que o grau de ajuste do modelo não-linear é substancialmente melhor do que o do modelo linear, cujo $R^{2}$ foi de 0,3681 . É necessário ressaltar o problema de variável omitida, ou de má especificação do modelo: sendo o modelo verdadeiro o não-linear, então se estaria omitindo a variável threshold no modelo linear, o que retornaria estimativas enviesadas (talvez responsável pelos pequenos betas dos estudos anteriores). Além disso, todos os coeficientes são estatisticamente significantes. Em ambos os regimes a hipótese de convergência é validada, $\hat{\alpha}_{1}<0$, e as velocidades de convergência e meias-vidas também estão mais em linha com as

\footnotetext{
${ }^{15} \mathrm{O} p$-valor de bootstrap foi gerado com base em 1000 replicações do modelo; utilizou-se um nível de significância de 5\% e fixou-se o corte mínimo amostral em $10 \%$ da amostra.
} 
encontradas na literatura: para o primeiro regime temos $\hat{\beta}_{I}=2,44 \%$ e $v\left(\hat{\beta}_{I}\right)=28$ anos, enquanto para o segundo regime tem-se $\hat{\beta}_{I I}=1,12 \%$ e $v\left(\hat{\beta}_{I I}\right)=62$ anos.

Os resultados sugerem uma maior velocidade de convergência para os estados que partem de uma renda per capita inicial mais elevada, sendo importante notar que esta modelagem permite identificar a formação de clubes de convergência, assim como convergência condicional: como há diferentes interceptos e diferentes inclinações para os regimes, então o diagrama no plano $\left[\frac{k_{t}^{*}}{k_{t}}, k_{t}\right]$ no modelo de Solow sugere duas posições de estado estacionário.

Aqui cabem alguns adendos em relação à robustez dos resultados encontrados, principalmente em relação à qualidade dos dados e das estimativas. Primeiramente, o pool de dados foi construído no intuito de se contornar o problema de poucas observações cross-section (20 regiões). Há algumas maneiras distintas de se agrupar dados: o pool poderia ter sido construído, por exemplo, com as taxas médias de crescimento para períodos mais curtos de tempo, digamos, de cinco em cinco anos, e suas respectivas rendas per capitas iniciais. O problema é que, ao se fazer isto, pode-se estar observando taxas de crescimento que não as de longo prazo, o que não é muito condizente com a ideia de regressões de crescimento. Neste caso, seria mais apropriado se utilizar painéis, como, por exemplo, foi proposto em Islam (1995), Caselli et alii (1996) e Bond et alii (2001). A metodologia de painel dinâmico vem sendo amplamente empregada para se estudar $\beta$-convergência e seria condizente com a análise aqui traçada utilizar um modelo de painel dinâmico com efeito threshold. Entretanto, a ciência estatística e econométrica ainda não avançaram o suficiente para que tal metodologia seja empregada, mas, conforme a mesma avance, esta promete ser uma interessante linha de pesquisa. ${ }^{16}$ Assim, seguiu-se a sugestão de Barro e Sala-I-Martin (1991) e optou-se por tomar períodos mais longos quanto possíveis na construção do pool.

Ademais, conforme foi discutido anteriormente, o pool de dados parece estar muito bem alinhado com as teorias e estudos empíricos dominantes, ou seja, a teorias de crescimento neoclássica e as análises empíricas nacionais que discorrem sobre formação de clubes de convergência no Brasil. Isto pode ser visto nas Figuras 3 e 4 do Apêndice. A Figura 3 plota as taxas médias de crescimento contra 0 logaritmo neperiano das rendas per capitas iniciais de cada estado levando-se em consideração o pool de dados construído. Foi feita uma distinção entre os estados que compõem o Regime I - marcados com quadrados cinza $^{17}$ — dos estados que compõem o Regime II — marcados com triângulos pretos. Na Figura, embora as retas da regressão não tenham sido traçadas, os coeficientes estimados da Tabela 2 deixam nítido que os estados do primeiro grupo, ou seja, os estados que detém renda per capita inicial mais elevada, deveriam vir se deslocando para posições de estado estacionário com renda per capita final também mais elevada, quando comparados aos estados do segundo grupo. A maior proximidade do eixo horizontal de uma parte dos estados com renda mais elevada, pertencentes ao Regime I (São Paulo, Rio de Janeiro e Rio Grande do Sul), também sugere que estes estados estão mais próximos de sua renda per capita de steady-state, o que também está de acordo com as meias-vidas encontradas. Para que se afirme isto com maior precisão também é necessário constatar que as taxas médias de crescimento dos estados vieram se reduzindo com o tempo. ${ }^{18}$ Isso pode ser visto na Figura 4, o qual retorna as densidades de kernel das taxas médias de crescimento para os períodos iniciais (1970-2003) e finais (1975-2008) do pool de dados, assim como as taxas de um período intermediário (1972-2005). ${ }^{19}$

\footnotetext{
${ }^{16}$ Shin (2008) já prospecta alguns avanços neste sentido, entretanto, seu trabalho ainda não foi concluído.

${ }^{17}$ Os estados marcados com quadrados cinza são os da região Amazônica ( $\left.\mathrm{AM}+\mathrm{AC}+\mathrm{RR}+\mathrm{RO}\right)$ e o estado da Bahia. Eles foram assim marcados para que se contraste os resultados obtidos através da metodologia de Hansen (2000) com os resultados advindos da metodologia de Phillips e Sul (2007).

${ }^{18}$ Ressalte-se que esse padrão dinâmico de redução das taxas de crescimento se mantém independentemente do período intermediário selecionado

${ }^{19} \mathrm{~A}$ ideia deste exercício se assemelha a identificar se as médias móveis das taxas de crescimento dos estados vêm declinando com o tempo
} 
A análise das densidades sugere que a distribuição das taxas de crescimento vieram se deslocando para a esquerda com o passar do tempo e que uma possível bi-modalidade vem sendo desfeita. Isso nos dá fortes indícios de que a estrutura do pool de dados está em fina sintonia com os modelos teóricos Neoclássicos.

Ao se trabalhar com dados agrupados, Lee et alii (1998) e Maddala e Wu (2000) mostram que a imposição a priori de restrições de homogeneidade pode enviesar as estimativas da $\beta$-convergência. Lee et alii (1998), por exemplo, reforçam que a validade das estimativas depende criticamente da presença da hipótese de taxas de crescimento comuns entre economias e que as estimativas com base no pool de dados seriam inconsistentes, mesmo com $N$ e $T$ grandes. No que se concerne a isso, é necessário ressaltar que o uso do efeito threshold tende reduzir substancialmente a heterogeneidade da amostra, pois tal efeito tende a segregar as economias, cada qual em seu regime, de acordo com suas características em termos de crescimento econômico. Neste sentido, é de se esperar que tal questão não contamine demasiadamente a análise.

Outro ponto de interesse é que neste trabalho não se está interessado em estimativas precisas do parâmetro $\beta$, daí a não inclusão de variáveis de controle. Nosso objetivo principal é apenas constatar se a $\beta$-convergência ocorre, ou não, e de que maneira é possível se conciliar a possível ocorrência deste fenômeno com a ocorrência da $\sigma$-convergência. Ademais, o modelo com efeito threshold pode ser pensado como um modelo em forma reduzida utilizado para capturar os efeitos destas variáveis condicionantes omitidas.

Por fim, é necessário observar que o teste do modelo com efeito threshold contra o modelo linear inicial é feito com base em replicações de bootstrap, o que garante um melhor grau de ajuste aos dados em amostras finitas.

\subsection{Phillips e Sul (2007)}

Formulando o coeficiente de transição relativa, $\left(\frac{H_{1}}{H_{t}}\right)$, e descartando-se os 13 primeiros anos para o cômputo das estimativas, conforme recomendado em Phillips e Sul (2007), a aplicação do teste $\log t$ para o painel de dados condensados dos PIBs per capita Estaduais retorna um coeficiente estimado $\hat{\pi}_{1}=-0.227$ com estatística $t_{\hat{\pi}}=-5.65184$; como este valor é menor do que -1.65 , este resultado sugere que não há convergência global dos níveis dos PIBs per capita. Há, entretanto, espaço para a divergência de alguma unidade ou a possível formação de clubes de convergência. Ao se aplicar sequencialmente o teste $\log t$, conforme descrito na subseção 3.2, a análise sugere que vêm se formando dois clubes de convergência no Brasil. Os estados que compõem cada um dos clubes e as estatísticas de teste $\log t$ associadas à regressão do teste são descritas na Tabela 3 , a seguir.

O primeiro grupo é essencialmente formado pelas economias do centro-sul enquanto o grupo restante é formado pelas economias do norte-nordeste. Posto que a estatística $t_{\hat{\pi}_{1}}$ é maior do que 1.65 para o grupo remanescente, também se tem evidências de que este grupo deve vir, de fato, convergindo para uma mesma renda per capita de longo prazo.

Note-se que a segregação resultante aqui é muito semelhante aos clubes de convergência identificados através da metodologia de Hansen (2000), a não ser pela Região Amazônica e pelo Estado da Bahia, que agora foram incorporados no grupo de renda mais abastada.

Ao contrário do teste proposto por Hansen (2000), é necessário ressaltar que o teste proposto por Phillips e Sul (2007) admite fenômenos como catch-ups e pushing-backs. Assim, estes resultados quando comparados aos da análise anterior parecem sugerir que, embora a Região Amazônica e o Estado da Bahia tenham ensaiado uma espécie de catch-up para o grupo de renda mais elevada, os mesmos retrocederam posteriormente e retornaram definitivamente para o grupo de renda mais baixa, conforme fica evidente na análise da dinâmica de transição para o estado estacionário, na Figura 2 do Apêndice.

As dinâmicas de transição dos grupos, cada qual para sua respectiva posição de estado estacionário auxilia na compreensão do porque a região Amazônica e o estado da Bahia diferem em relação aos 
Tabela 3: O teste de Phillips e Sul (2007) para 1970-2008

\begin{tabular}{|c|c|c|c|}
\hline \multicolumn{4}{|c|}{$\log \frac{H_{1}}{H_{t}}-2 \log [L(t)]=\pi_{0}+\pi_{1} \log t+u_{t}^{20}$} \\
\hline GRUPO 1 & Parâmetro & $\hat{\pi}$ & $t_{\hat{\pi}}$ \\
\hline Espírito Santo, (Goiás + Tocantins), Minas Gerais, (Mato & $\pi_{0}$ & $-3,953$ & $-27,115$ \\
\hline $\begin{array}{l}\text { Grosso + Mato Grosso do Sul), Paraná, Rio de Janeiro, Rio } \\
\text { Grande do Sul, Santa Catarina e São Paulo. }\end{array}$ & $\pi_{1}$ & 0,850 & 18,936 \\
\hline GRUPO 2 & Parâmetro & $\hat{\pi}$ & $t_{\hat{\pi}}$ \\
\hline Alagoas, (Amazonas + Acre + Rondônia + Roraima), Bahia, & $\pi_{0}$ & $-3,922$ & $-7,905$ \\
\hline $\begin{array}{l}\text { Ceará, Maranhão, (Pará + Amapá), Paraíba, Pernambuco, } \\
\text { Piauí, Rio Grande do Norte, e Sergipe. }\end{array}$ & $\pi_{1}$ & 0,473 & 3,099 \\
\hline
\end{tabular}

Fonte: Elaboração do autor.

grupos identificados pelas metodologias ora empregadas: estas duas unidades possuíam alta renda per capita inicial e as dinâmicas de suas rendas per capitas parecem sugerir o referido ensaio de catch-up até o final de 1986, sendo que, posteriormente, tal ensaio finda e estas unidades voltam a convergir para a posição de estado estacionário do clube de renda mais baixa.

A análise gráfica da dinâmica de transição dos clubes de convergência identificados sugere uma nítida ocorrência de $\sigma$-convergência "intra-clube", assim como este tipo de convergência para o primeiro clube parece ser mais provável, pois se observa uma redução da dispersão em torno da média relativamente mais intensa para este grupo do que para o segundo.

Em face à dimensão do painel de dados utilizado, 38 observações no tempo $(T)$ para 20 unidades transversais $(N)$, é importante avaliar as propriedades dos testes e procedimentos aplicados em pequenas amostra, já que eles são baseados em aproximações assintóticas. Essa avaliação, realizada por meio de simulações de Monte Carlo, é apresentada no apêndice do próprio artigo de Phillips e Sul (2007). Primeiramente, os autores destacam que o tamanho e o poder do teste $\log t$ estão atrelados à fração da amostra inicial a ser descartada, $r$, e à função slowling varying utilizada, $L(t)$. Para um painel com $T=50$ e $N=25$, dimensão mais próxima da utilizada no presente estudo, os autores sugerem que $o$ descarte de um terço da amostra inicial $(r=0,3)$ e o uso de $L(t)=\log t$ são as opções que geram melhores resultados em termos de redução do erro do tamanho do teste e aumento do poder. Eles mostram que o poder do teste $\log t$ passa de aproximadamente 0,5 , para valores de $\alpha$ menores do que 0,2 para 0,97 quando $\alpha$ é maior do que 0,4 (ou seja, para pequenos valores de $\alpha$, existe uma tendência de não rejeitar convergência quando ocorre divergência). Isso piora quando $r$ cresce. Em termos de tamanho, quando $\alpha \leq 0,2$, o tamanho real do teste é bem próximo do nominal (5\%). Entretanto, o tamanho do teste se torna mais conservativo, com taxa nominal em torno de $1 \%$, quando $\alpha \geq 0,4$ (ou seja, existe uma leve tendência de não rejeitar a hipótese de convergência). Pelas estimativas de $\alpha$, em torno de -0.113 para o grupo geral, de 0,425 para o GRUPO 1 e 0,237 para o GRUPO 2, esses problemas não são tão graves aqui. Observe, no entanto, que o resultado mais esperado no caso do grupo geral, que seria a não rejeição de convergência, não se consolidou. ${ }^{21}$ Assim, considerando de uma forma geral a avaliação dos autores, pode-se assegurar certa confiabilidade dos resultados quanto à escolha de $r$ e $L(t)$.

\footnotetext{
${ }^{21}$ Por outro lado, pelo menos em relação ao poder do teste, poderia ser questionado se o GRUPO 2 estaria de fato convergindo. Note-se, no entanto, que o tamanho do teste é provavelmente correto neste caso.
} 
Em termos de viés, os experimentos sugerem que as estimativas de $b$, que define a tendência para a qual as unidades estão convergindo, são um pouco menores do que seus verdadeiros valores. Eles mostram ainda que esse viés negativo é mais dependente do tamanho de $T$ e de $\alpha$ do que do tamanho de $N$, e que o mesmo se dissipa rapidamente para valores de $\alpha$ e/ou $T$ relativamente grandes.

Phillips e Sul (2007) também investigam o desempenho do procedimento de deteç̧ão de clubes e seleção dos integrantes pertencentes aos mesmos, o qual é definido pelo algoritmo de agrupamento descrito na subseção 3.2. No caso de divergência ou formação de clubes, o poder do teste é alto, aproximadamente 1 para o caso $T=40$ e $N=50$ e 0,93 para $T=10$ e $N=50$, independente dos valores de $\alpha$. Entretanto, o poder é bastante reduzido quando, sob a hipótese alternativa, os parâmetros de convergência que tornam os clubes distintos são semelhantes; ou seja, quando $b_{1}$ é bem próximo de $b_{2}$.

Quanto ao algoritmo de agrupamento, Phillips e Sul sugerem que, em amostras finitas, conforme se emprega valores críticos mais amplos no procedimento de seleção dos integrantes dos clubes (ver definição do critério de fixação, $c^{*}$, na discussão do algoritmo), mais poder o teste passa a ter. Além disso, este ganho de poder em se utilizar um nível de significância maior excede a perda de tamanho do teste. O experimento de Monte Carlo de Phillips e Sul também sugere que a acurácia na seleção dos membros dos clubes apresenta melhor desempenho quando se observa valores de $\alpha$ relativamente grandes. Para se checar a robustez dos resultados para $T$ relativamente pequeno os autores sugerem que se fixe $c^{*}=0$ e que se observe se há alteração na formação dos clubes. Seguindo-se tal estratégia, não se observa alteração nos clubes, o que sugere que o nosso resultado do agrupamento dos membros dos clubes parece ser robusto.

\section{CONCLUSÕES}

A proposta de que a renda per capita de diferentes economias deveria convergir para um patamar comum é um resultado direto do modelo de Solow (1956). ${ }^{22}$ A partir da "equação fundamental da dinâmica do capital", tal modelo foi pioneiro em sugerir que, uma vez que a tecnologia e a taxa de poupança fossem semelhantes entre as economias, a produtividade marginal do capital decrescente cessaria o crescimento no longo prazo e, por conseguinte, as economias convergiriam para um mesmo nível de renda per capita.

Num contexto regional, com ampla mobilidade de capital e barreiras relativamente baixas à migração, o estudo de Barro e Sala-I-Martin (2004) para países europeus, estados norte americanos e prefeituras do Japão parece sugerir que a $\beta$-convergência e a $\sigma$-convergência devessem ocorrer em conjunto, entretanto, Young et alii (2008) sustentam que para os condados norte americanos essa hipótese tende a ser violada.

No que concerne à literatura empírica que tratou do tema para o Brasil, a mesma sugere que, assim como na análise de Young et alii (2008), os resutados para estas duas análises são aparentemente conflitantes: enquanto os exames cross-section de $\beta$-convergência sugerem que este tipo de convergência ocorre, as análises de séries temporais da $\sigma$-convergência não ratificam essa evidência.

Aqui, acredita-se que este desconforme se deve, provavelmente, à negligência do processo de formação de clubes de convergência nestas análises e, com o intuito de investigar essa controvérsia mais a fundo, propõe-se que se investigue se a mesma permanece ocorrendo intra-clubes. ${ }^{23}$

Após a formulação deste ponto, duas metodologias para testar a hipótese de convergência levando em consideração uma possível não-linearidade capaz de acomodar a presença de clubes de convergência foram apresentadas: a primeira foi proposta por Hansen (2000), que é um teste cujo foco repousa sobre

\footnotetext{
${ }^{22}$ Esta premissa pode ser estendida a diversos outros modelos como, por exemplo, Barro e Sala-I-Martin (1997) e Howitt (2000).

${ }^{23} \mathrm{O}$ processo de formação de clubes de convergência sugere que o diferencial entre os níveis de renda dos clubes pode se ampliar com o passar do tempo e este diferencial possivelmente está associado a diversas questões. Análises teóricas sustentam que (i) a educação (Azariadis e Drazen, 1990), (ii) o crédito (Aghion et alii, 2005), (iii) a infra-estrutura (Barro, 1990), as instituições (Acemoglu, 2008) e, (iv) a desigualdade social (Barro, 2000), podem ser uma destas questões.
} 
regressões cross-section e está intimamente ligado à análise de $\beta$-convergência e; a segunda é uma análise de séries temporais sugerida por Phillips e Sul (2007) que, por sua vez, pode ser caracterizada como uma análise de $\sigma$-convergência. Em resumo, ambos os testes podem ser entendidos como análises incondicionais de convergência (no sentido de que não necessitam de variáveis explicativas adicionais para controlar para diferentes posições de estado estacionário).

Após a apresentação dos dados utilizados na análise empírica (um painel que reúne dados de Azzoni (1997) para 1970-1985 e dados do IPEA para 1985-2008), os testes de Hansen (2000) e Phillips e Sul (2007) são conduzidos para os PIBs per capitas estaduais. Ambos os testes indicam formação de clubes de convergência, além da conhecida segregação regional entre os estados do centro-sul e os estados do norte-nordeste.

A abordagem da $\sigma$-convergência (Phillips e Sul, 2007) sugere a formação de um clube de convergência cuja renda per capita é mais elevada, formado pelos estados do Espírito Santo, Goiás + Tocantins, Minas Gerais, Mato Grosso + Matogrosso do Sul, Paraná, Rio de Janeiro, Rio Grande do Sul, Santa Catarina e São Paulo; e outro formado pelos demais estados cuja renda per capita é relativamente menor do que a do primeiro. A abordagem da $\beta$-convergência (Hansen, 2000) também sugere a formação de dois clubes de convergência muito próximos dos identificados anteriormente, entretanto, sob tal proposta, a Bahia e a Região Amazônia (AM, AC, RO, RR) fariam parte do clube de renda per capita mais alta.

A leve distorção entre estas duas metodologias parece estar associada a um fenômeno de pushingback, sendo necessário ressaltar que a metodologia de Phillips e Sul (2007) é capaz de acomodar fenômenos como este, mas a de Hansen (2000) não. Embora Bahia e Amazônia tenham tido um bom desempenho no período inicial da análise, este desempenho parece não ter sido suficientemente forte para que os mesmos se descolassem do segundo grupo e acompanhassem as trajetórias das rendas per capita dos estados mais ricos.

Embora a qualidade dos dados estaduais brasileiros possa ser questionada, tanto para as análises de séries de tempo quanto para as análises cross-section, os resultados aqui reportados sugerem que $o$ uso de modelos não-lineares pode ser mais apropriado para os dados, além de dirimir a controvérsia empírica encontrada entre os teste de $\sigma$ e $\beta$-convergência.

Laurini et alii (2005) fazem uso de dados municipais e também aplicam métodos econométricos capazes de acomodar efeitos não lineares. ${ }^{24}$ Este último estudo corrobora nossa análise indicando que há a formação de clubes de convergência e que se tem evidencia tanto de $\sigma$ quanto de $\beta$-convergência.

Em resumo, o que parece ocorrer é o seguinte: Dois clubes estão se formando no Brasil o centro-sul e o norte-nordeste. Regressões cross-section para a média, desconsiderando a formação destes clubes, ainda validam a hipótese da $\beta$-convergência; entretanto, análises de séries temporais que assim o fazem parecem violar a hipótese de $\sigma$-convergência. Assim, uma aparente controvérsia entre análises de $\sigma \mathrm{e}$ $\beta$-convergência se verifica.

A razão para isto é que as diferentes condições estruturais das economias tendem a levar as mesmas para posições de steady-state distintas. Respeitando-se tais posições, ou seja, investigando-se o processo de convergência intra-clubes, observa-se que tanto a $\sigma$ quanto a $\beta$-convergência ocorrem.

A questão dos clubes de convergência parece ser um ponto central na análise e, ao se levar em conta a mesma, a controvérsia entre análises de $\sigma$ e $\beta$-convergência tende a se dirimir. Conectando nossos resultados com os estudos já feitos para o Brasil, parece haver fortes indícios de que esteja

\footnotetext{
${ }^{24}$ Uma classe importante de métodos econométricos que permitem capturar a existência de efeitos não-lineares, tanto para $\sigma$ quanto para $\beta$-convergência, são as análises baseadas em métodos não-paramétricos, como as análises baseadas em dinâmicas de transição estimadas por kernel density, como utilizadas no próprio artigo de Quah (1996), e as regressões não-paramétricas (Nadaraya-Watson, Local Polynomial, etc), que foram métodos utilizados na análise de Liu e Stengos (1999). Para analisar $\sigma$ convergência e $\beta$-convergência, Laurini et alii (2005) utilizaram testes baseados no princípio do Bootstrap em Smoothing Splines, respectivamente. Apesar desta classe de estimadores apresentarem vantagens sobre os estimadores paramétricos como os utilizados aqui, a mesma faz uso de matrizes de transição. Utilizando uma estrutura de dados semelhante a que foi utilizada aqui, Cabral (2008) revela que a matriz de transição parece não ser estatisticamente estável, o que torna tais técnicas inapropriadas para analise, pelo menos em se tratando de dados estaduais. Incentivamos, entretanto, investigações empíricas nesse sentido.
} 
havendo uma polarização das rendas per capitas estaduais, ou seja, há indícios de que o diferencial entre as rendas per capitas estaduais esteja se ampliando com o tempo. Isto decorre do fato de que, embora haja constatação de $\sigma$-convergência intra-clubes, os estudos anteriores tendem a descartar esta hipótese para toda amostra.

\section{BIBLIOGRAFIA}

Abitante, K. G. (2007). Desigualdade no Brasil: Um estudo sobre convergência de renda. Pesquisa E Debate, 18(2):155-169.

Abramovitz, M. (1986). Catching up, forging ahead, and falling behind. Journal of Economic History, 46(2):385-406.

Acemoglu, D. (2008). Introduction to modern economic growth. Technical report, Department of Economics. MIT. Mimeo.

Aghion, P., Angeletos, P.-M., Banerjee, A., \& Manova, K. (2005). Volatility and growth: Credit constraints and productivity-enhancing investment.

Andrade, E., Laurini, M., \& Vals Pereira, P. L. (2004). Clubes de convergência de renda para os municípios brasileiros: Uma análise não-paramétrica. Working Papers 6, IBMEC.

Azariadis, C. \& Drazen, A. (1990). Threshold externalities in economic development. The Quarterly Journal of Economics, 105(2):501-526.

Azzoni, C. (1997). Concentração regional e dispersão das rendas per capita estaduais: Análise a partir de séries históricas estaduais de pib 1939-1995. Estudos Econômicos, 27(3):341-393.

Azzoni, C. R. (2001). Economic growth and regional income inequalities in Brazil (1939-1992). The Anals of Regional Science, 35:133-152.

Azzoni, C. R., Menezes-Filho, N., Menezes, T., \& Silveira-Neto, R. (2000). Geography and income convergence among Brazilian states. Research Network Working Paper R-395, Inter-American Development Bank, Washington DC.

Barossi-Filho, M. \& Azzoni, C. R. (2003). A time series analysis of regional income convergence in Brazil. TD Nereus TD 09-2003.

Barro, R. (1990). Government spending in a simple model of endogeneous growth. Journal of Political Economy, 98(S5):103-125.

Barro, R. (2000). Inequality and growth in a panel of countries. Journal of Economic Growth, 5(1):5-32.

Barro, R. \& Sala-I-Martin, X. (1991). Convergence across states and regions. Brookings Papers on Economic Activity 1, pages 107-182.

Barro, R. J. \& Sala-I-Martin, X. (1997). Technological diffusion, convergence and growth. Journal of Economic Growth, 2(1):1-26.

Barro, R. J. \& Sala-I-Martin, X. (2004). Economic Growth. McGraw-Hill, Inc.

Basu, S. \& Weil, D. (1998). Appropriate technology and growth. Quarterly Journal of Economics, 113:10251054. 
Baumol, J, W. (1986). Productivity growth, convergence, and welfare: What the long-run data show. The American Economic Review, 76(5):1072-1085.

Bernard, A. B. \& Durlauf, S. N. (1995). Convergence in international output. Journal of Applied Econometrics, 10:97-108.

Beyaert, A. \& Camacho, M. (2008). Tar panel unit root tests and real convergence. Review of Development Economics, 12:607-620.

Bond, S., Hoeffler, A., \& Temple, J. (2001). GMM estimation of empirical growth models. Technical report, University of Oxford, Institute for Fiscal Studies.

Cabral, E. C. S. (2008). Convergência de renda per capita entre os Estados brasileiros de 1939 a 2004. Tese de doutorado em economia, Universidade de Brasília.

Cangussu, R. C., Salvato, M. A., \& Nakabashi, L. (2010). Uma análise do capital humano sobre o nível de renda dos estados brasileiros: MRW versus Mincer. Estudos Econômicos, 40(1):153-183.

Caselli, F., Esquivel, G., \& Lefort, F. (1996). Reopening the convergence debate: A new look at cross country growth empirics. Journal of Economic Growth, 1(3):363-389.

Chaves, M. C. (2003). Examinando as desigualdades regionais: Um teste de convergência para a renda per capita familiar brasileira, 1970-1991. Dissertação apresentada ao programa de mestrado em desenvolvimento econômico, Universidade Federal do Paraná.

Coelho, R. L. \& Figueiredo, L. (2007). Uma análise da hipótese da convergência para os municípios brasileiros. Revista Brasileira de Economia, 61(3):331-351.

Costa, L. M. (2009). Análise do processo de convergência de renda nos estados brasileiros: 1970-2005. Dissertação de mestrado, Fundação Getúlio Vargas.

Dos Santos, C. M. \& Carvalho, F. M. A. (2007). Dinâmica das disparidades regionais da renda per capita nos estados brasileiros: Uma análise de convergência. Revista Economia e Desenvolvimento, 19.

Durlauf, S. N. \& Johnson, P. A. (1995). Multiple regimes and cross-country growth behavior. Journal of Applied Econometrics, 10(4):365-384.

Durlauf, S. N., Johnson, P. A., \& Temple, J. R. W. (2004). Growth econometrics. Working Papers 61, Vassar College.

Ellery JR., R. \& Ferreira, P. C. (1994). Crescimento econômico e convergência entre as rendas dos estados brasileiros. In Anais do XVI Encontro Brasileiro de Econometria (SBE), pages 264-286.

Evans, P. \& Karras, G. (1996). Convergence revisited. Journal of Monetary Economics, 37:249-265.

Ferreira, A. H. B. (1998). Evolução recente das rendas per capita estaduais no Brasil. Revista de Economia Política, 18:90-97.

Ferreira, A. H. B. (1999). Concentração regional e dispersão das rendas per capita estaduais: Um comentário. Estudos Econômicos, 29(1):47-63.

Ferreira, A. H. B. (2000). Convergence in Brazil: Recent trends and long-run prospects. Applied Economics, 32:479-489.

Ferreira, A. H. B. \& Diniz, C. (1995). Convergência entre as rendas per capita estaduais no Brasil. Revista de Economia Política, 15(4):38-56. 
Ferreira, A. H. B. \& Diniz, C. (1996). A distribuição interestadual da renda no Brasil (1950-85). Revista Brasileira de Economia, 50:469-485.

Fleming, J. M. (1955). External economies and the doctrine of balanced growth. Economic Journal, 65:241-256.

Furceri, D. (2005). [beta] and [sigma]-convergence: A mathematical relation of causality. Economics Letters, 89(2):212-215.

Galor, O. \& Weil, D. N. (2000). Population, technology, and growth: From the Malthusian regime to the demographic transtition and beyond. American Economic Review, 90:806-828.

Galor, O. \& Zeira, J. (1993). Income distribution and macroeconomics. Review of Economic Studies, 60(1):35-52.

Gondim, J. L. B., Barreto, F. A., \& Carvalho, J. R. (2007). Condicionantes de clubes de convergência no Brasil. Estudos Econômicos, 37(1):71-100.

Hansen, B. E. (1996). Inference when a nuisance parameter is not identiffed under the null hypothesis. Econometrica, 64:413-430.

Hansen, B. E. (2000). Sample splitting and threshold estimation. Econometrica, 68:575-603.

Howitt, P. (2000). Endogenous growth and cross-country income differences. The American Economic Review, 90(4):829-846.

Howitt, P. \& Mayer-Foulkes, D. (2005). R\&D, implementation and stagnation: A Schumpeterian theory of convergence clubs. Journal of money, credit and Banking, 37:147-177.

Huang, H.-C. (2005). Diverging evidence of convergence hypothesis. Journal of Macroeconomics, 27(2):233-255.

Islam, N. (1995). Growth empirics: A panel data approach. Quarterly Journal of Economics, 110(4):11271170.

Johnson, P. \& Takeyama, L. (2003). Absolute, conditional or club convergence in the U.S. states? Vassar College. Mimeografado.

Laurini, M., Andrade, E., \& Valls Pereira, P. (2005). Income convergence clubs for Brazilian municipalities: A non-parametric analysis. Applied Economics, 37:2099-2118.

Lee, K., Pesaran, H., \& Smith, R. (1998). Growth and convergence in a multi-country empirical stochastic Solow model. Journal of Applied Econometrics, 112:357-392.

Lewis, A. (1956). Is the AK model still alive? The long-run relation between growth and investment reexamined, volume 35, Canadian Journal of Economic, The Theory of Economic Growth, pages 92-114. London: Allen \& Unwin. LI, D.

Lima, L. R., Notini, H. H., \& Gomes, F. A. R. (2010). Empirical evidence on convergence across Brazilian states. Revista Brasileira de Economia, 64(2).

Liu, Z. \& Stengos, T. (1999). Non-linearities in cross-country growth regressions: A semiparametric approach. Journal of Applied Econometrics, 14(5):527-538.

Lucas, R. (2000). Some macroeconomics for the 21st century. Journal of Economic Perspectives, 11:159168. 
Lucas, R. E. J. (2002). The industrial revolution: Past and future. In Lucas, R. E. J., editor, Lectures on Economic Growth: Cambridge. Massachusetts: Harvard University Press.

Maddala, G. S. \& Wu, S. (2000). A comparative study of unit root tests with panel data and a new simple test. Oxford Bulletin of Economics and Statistics, 61:631-652.

Maddison, A. (2004). The world economy: Historical statistics. OECD, Paris: Development Studies Centre.

Mankiw, G., Romer, P., \& Weil, D. N. (1992). A contribution to the empirics of economic growth. Quarterly Journal of Economics, 107:407-437.

Mello, M. (2011). Stochastic convergence across Brazilian states. Brazilian Review of Econometrics, 30:2352.

Mello, M. \& Guimarães-Filho, R. (2007). A note on fractional stochastic convergence. Economics Bulletin, 3:1-14.

Mossi, M. B., Aroca, P., Fernandez, I. J., \& Azzoni, C. R. (2003). Growth dynamics and space in Brazil. International Regional Science Review, 26(3):393-418.

Murphy, K., Shleifer, A., \& Vishny, R. (1989). Industrialization and the big push. Journal of Political Economy, 97:1003-1026.

Nurkse, R. (1953). Problems of Capital Formation in Underdeveloped Countries. New York: Oxford University Press.

Parente, S. L. \& Prescott, E. C. (1994). Barriers to technology adoption and development. Journal of Political Economy, 102:298-321.

Penna, C. M. \& Linhares, F. C. (2009). Convergência e formação de clubes no Brasil sob a hipótese de heterogeneidade no desenvolvimento tecnológico. Revista Econômica do Nordeste, 40(4).

Pesaran, M. H. (2007). A pair-wise approach to testing for output and growth convergence. Journal of Econometrics, 138(1):312-355.

Phillips, P. C. B. \& Sul, D. (2007). Transition modeling and econometric convergence tests. Econometrica, Econometric Society, 75(6):1771-1855.

Quah, D. (1996). Twin peaks: Growth and convergence in models of distribution dynamics. Economic Journal,, 106:1045-1055.

Resende, G. M. \& Figueiredo, L. (2005). Testes de robustez: Uma aplicação para os determinantes das taxas de crescimento do produto interno bruto per capita dos estados brasileiros. Texto para Discussão 1124, IPEA, Brasília.

Rosenstein-Rodan, P. (1943). Problems of industrialization of Eastern and South-Eastern Europe. Economic Journal, 53:202-211.

Rostow, W. W. (1960). The Stages of Economic Growth. Oxford University Press.

Scitovsky, T. (1954). Two concepts of external economies. Journal of Political Economy, 62:143-151.

Shin, Y. (2008). Bootstrap-based bias corrected within estimation of threshold regression models in dynamic panels. Technical report, Leeds University Business School. 
Silveira-Neto, R. \& Azzoni, C. R. (2005). Decomposing regional growth: Labor force participation rates, structural changes, and sectoral factor reallocation. The Annals of Regional Science, 39(2):221-239.

Solow, R. M. (1956). A contribution to the theory of economic growth. The Quarterly Journal of Economics, 70(1):65-94.

Stock, J. H. (1991). Confidence intervals for the largest autoregressive root in U.S. macroeconomic time series. Journal of Monetary Economics, 28(3):435-459.

Trompieri Neto, N., Castelar, I., \& Linhares, F. C. (2008). Convergência de renda dos estados brasileiros: Uma abordagem de painel dinâmico com efeito threshold. Anais do XXXVI Encontro Nacional de Economia [Proceedings of the 36th Brazilian Economics Meeting], ANPEC - Associação Nacional dos Centros de Pós-graduação em Economia [Brazilian Association of Graduate Programs in Economics].

Veloso, F. A., Villela, A., \& Giambiagi, F. (2008). Determinantes do "milagre" econômico brasileiro (19681973): Uma análise empírica. Revista Brasileira de Economia, 62(2):221-246.

Young, A. T., Higgins, M. J., \& Levy, D. (2008). Sigma convergence versus beta convergence: Evidence from U.S. county-level data. Journal of Money Credit And Banking, 40(5):1083-1093. 


\section{A. APÊNDICE}

Figura 2.1 - Dinâmicas de transição para os clubes

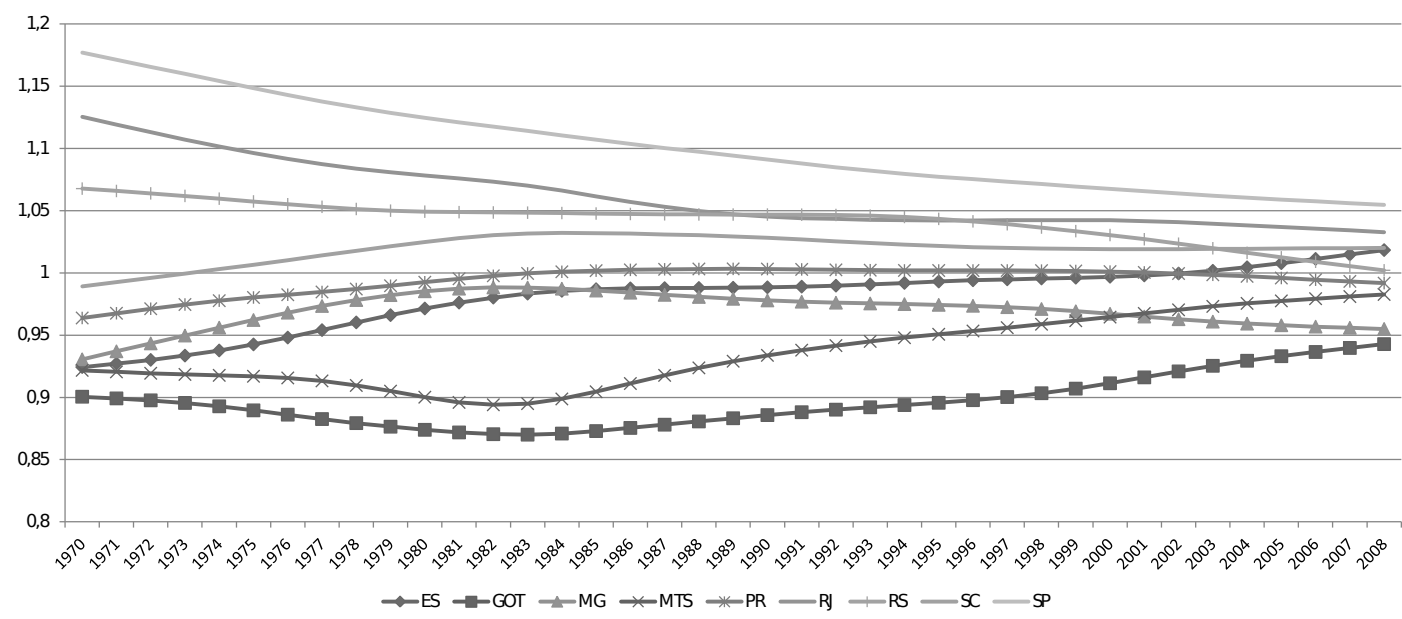

Figura 2.2

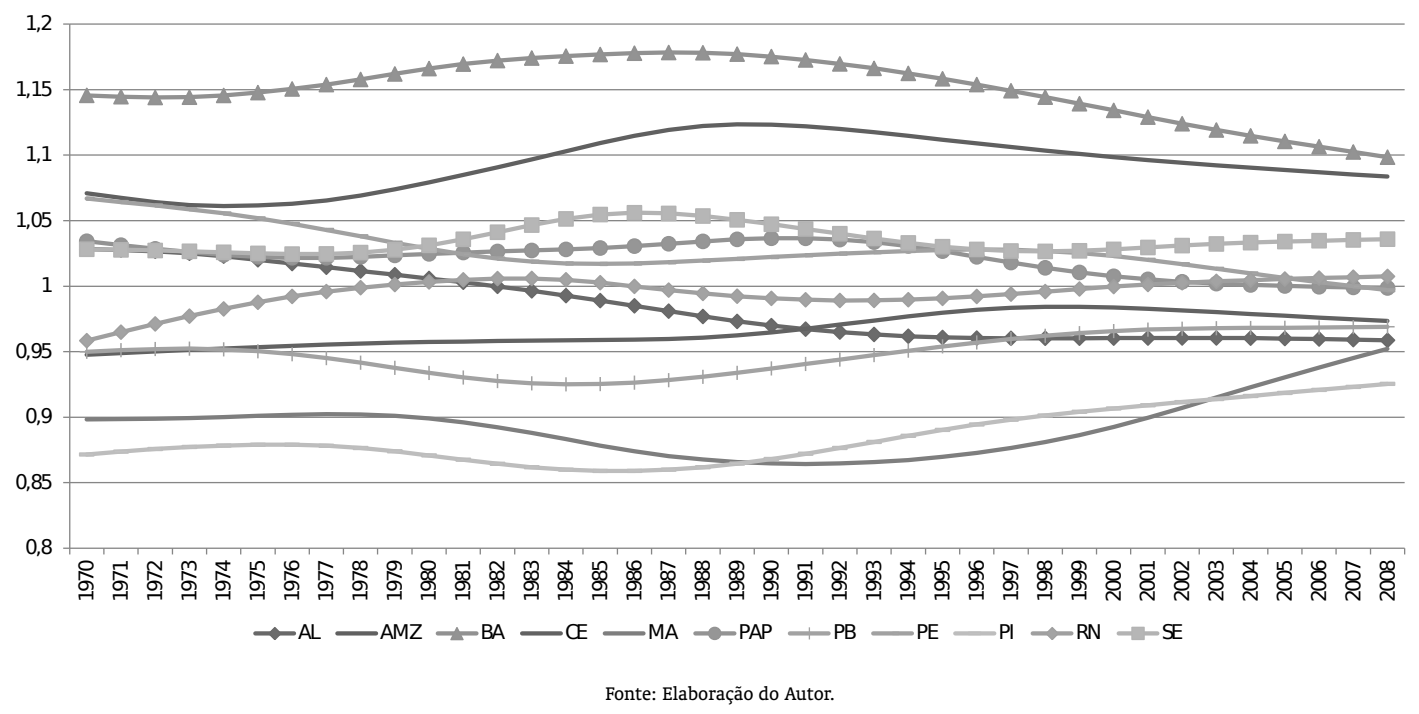

Nota: O eixo vertical dos gráficos representa $h_{i t}=\frac{\hat{w}_{i} t}{N^{-1} \sum_{i=1}^{N} \hat{w}_{i t}}$, onde $w_{i t}$ representa o logaritmo neperiano da renda per capita do estado $i$ após a remoção do componente de ciclos através do filtro de Hodrick-Prescott. 
Figura 3 - Pool de dados de taxas médias de crescimento contra log das rendas per capita iniciais, para 1970-2003, 1971-2004, 1972-2005, 1973-2006, 1974-2007, 1975-2008

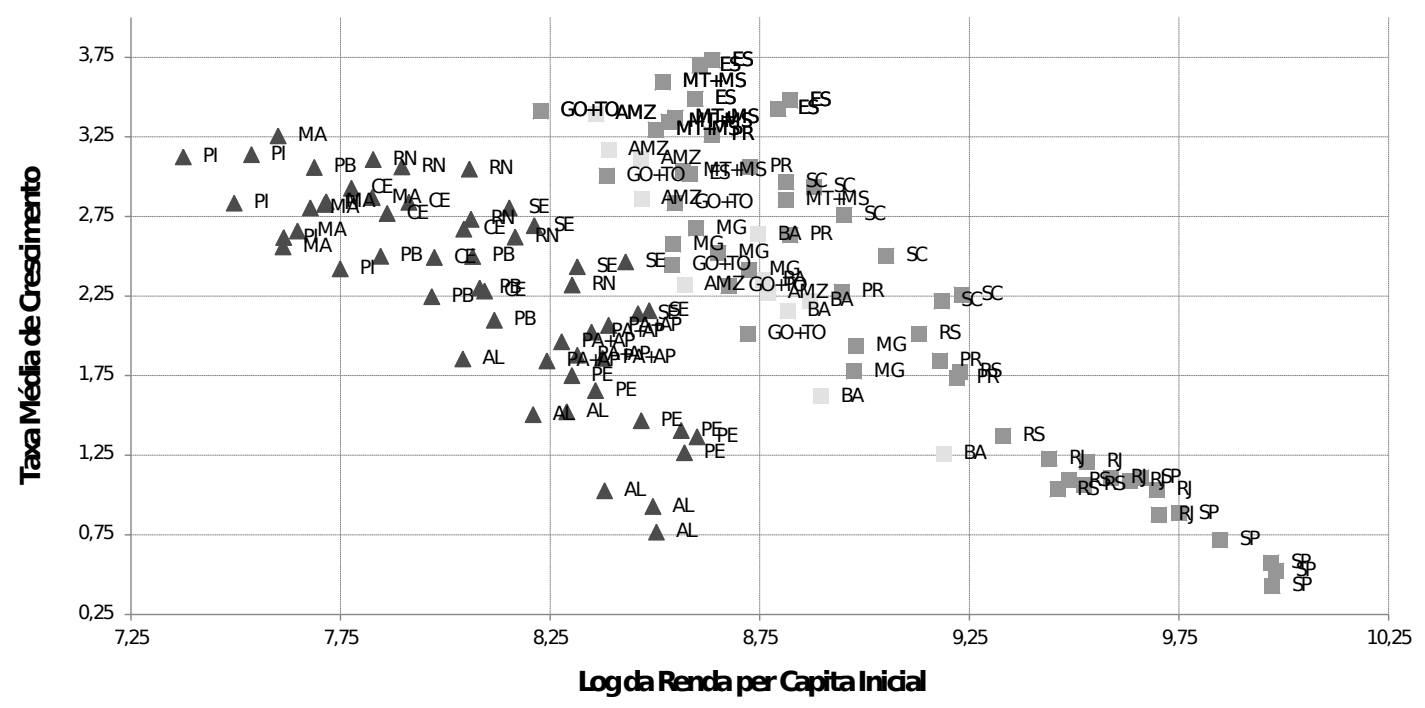

Figura 4 - Dinâmica da densidade de kernel das taxas médias de crescimento 1970-2003, 1972-2005 e 1975-2008

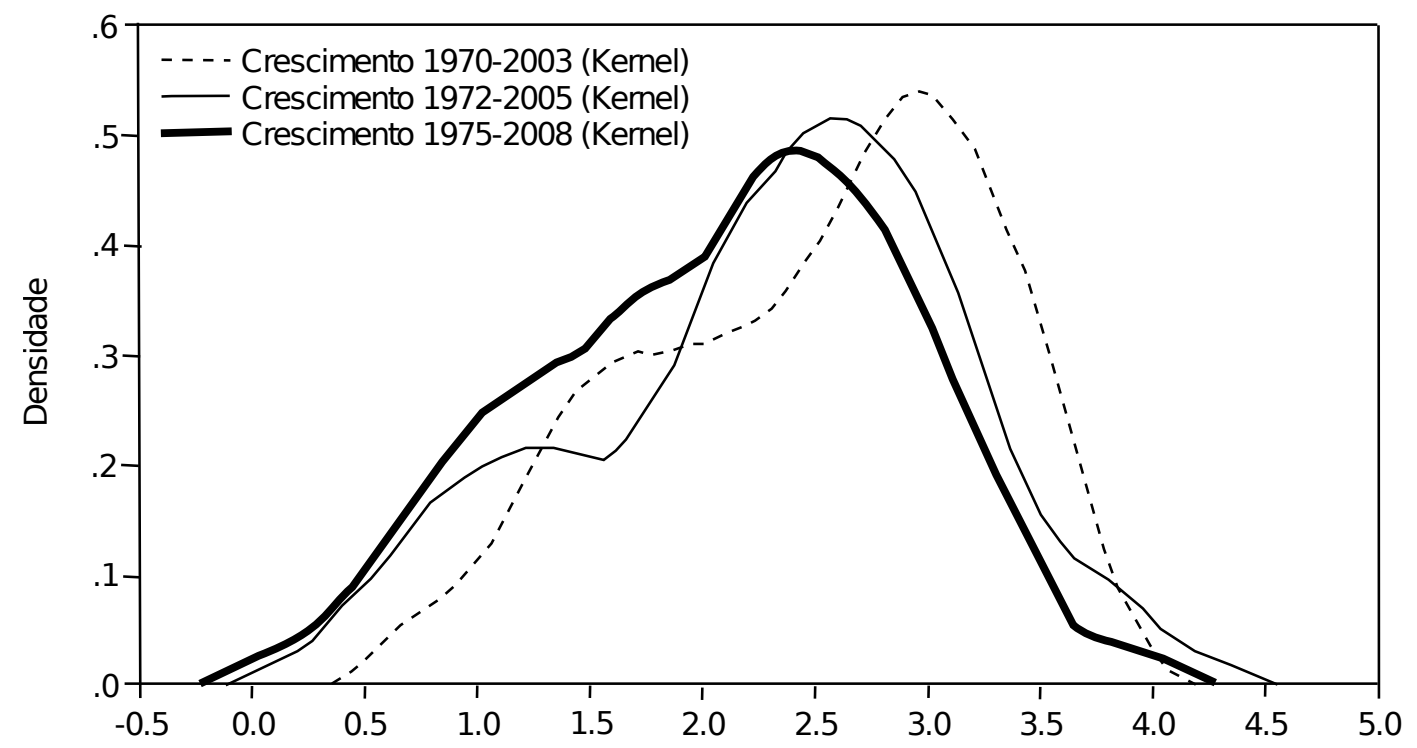

